\title{
Review: therapy based rehabilitation services reduce the risk of deterioration in patients who have had a stroke
}

Legg L, Langhorne P; Outpatient Service Trialists. Rehabilitation therapy services for stroke patients living at home: systematic review of randomised trials. Lancet 2004;363:352-6.

Outpatient Service Trialists. Therapy-based rehabilitation services for stroke patients at home. Cochrane Database Syst Rev 2003; (1):CD002925

\section{$Q$ In community dwelling patients who have had a stroke, do therapy based rehabilitation services offered $\leqslant 1$ year after stroke onset or discharge from hospital reduce deterioration in ability to perform activities of daily living (ADL)?}

\section{METHODS}

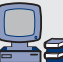
CINAHL, PsycLIT, EMBASE/Excerpta Medica, AMED, Social

Science Citation Index, Science Citation Index, bibliographies of relevant articles, and researchers in the field. Trial searching was completed in November 2001.

Study selection and assessment: randomised controlled trials (RCTs) that compared outpatient therapy based rehabilitation services (ie, interventions provided by qualified physiotherapists, occupational therapists, multidisciplinary staff, or under the supervision of qualified therapy staff) with no routine input. Quality of the RCTs was evaluated using criteria including method of generating the random sequence, concealment of allocation, masking of outcome assessment, and intention to treat analysis.

\begin{tabular}{|l|}
\hline \\
2 \\
3 \\
\hline
\end{tabular}

Outcomes: risk of deterioration in ability to perform ADL or becoming dependent in ADL by the end of scheduled follow up. Deaths were included as deterioration.

\section{MAIN RESULTS}

14 RCTs $(n=1617)$ met the selection criteria. Types of interventions included occupational therapy (8 RCTs), physiotherapy (2 RCTs), and multidisciplinary team services (4 RCTs). Meta-analyses were done using both fixed effects (for binary outcomes) and random effects (for continuous outcomes) models. Overall, the risk of deterioration was lower in the intervention group than the control group (table). The intervention group had higher (better) scores than the control group for ADL ( 12 RCTs, $\mathrm{n}=1180$; standardised mean difference [SMD] $0.14,95 \%$ CI 0.02 to 0.25 ) and extended ADL (9 RCTs, $\mathrm{n}=996$; SMD 0.17, 95\% CI 0.04 to 0.30 ).

\section{CONCLUSION}

In community dwelling patients who have had a stroke, therapy based rehabilitation services offered $\leqslant 1$ year after stroke onset or

For correspondence: Mrs L Legg, Glasgow University, Glasgow Royal Infirmary, Glasgow, UK. lynn@legg80.freeserve.co.uk

Sources of funding: UK Stroke Association and Chest Heart and Stroke Scotland. discharge from hospital reduce the risk of patient deterioration in ability to perform activities of daily living.

\section{Commentary}

The importance of evaluating the effectiveness of outpatient rehabilitation services for stroke patients is critical for effective use of healthcare budgets, anticipatory human resource planning, and targeted, timely care interventions. Therefore, the systematic review of RCTs by the Outpatient Service Trialists provides an excellent assessment of published and unpublished evidence on community based rehabilitation.

The results of this review, however, show modest health gains. To conclude with "What does seem clear is that the debate should move from whether such services are effective to how to make the most of their benefits" seems somewhat premature and overenthusiastic based on the numbers and the possibility, as acknowledged by the authors, that "... different groups of interventions might differ in their effects." Instead, the results suggest the need for future research to answer several questions: (1) What specific interventions (exact nature and content) are directly associated with preventing deterioration in personal ADL? (2) What are the treatment or interventional elements of the multidisciplinary team services (4 RCTs), and how do they contrast with the occupational therapy (8 RCTs) and physiotherapy (2 RCTs) services. The answers to these questions have implications for the role (if any) of nursing in outpatient rehabilitation services. (3) What are the implications for service or human resource planning, skill mix, scope (and overlapping scopes) of practice, cross training, and transferable skills sets? (4) What is the effect of "carers" (caregivers) given that some trials included only participants who had carers who were able to participate? (5) Are positive outcomes sustained after scheduled follow up or discharge from community programmes?

In short, this well done review highlights the need for continual examination of what is effective, how it is effective, and when it should be provided-questions commonly raised in relation to rehabilitation programmes. Further research is needed to provide firm conclusions on these points.

Heather M Campbell, RN, MS College of Nurses of Ontario Toronto, Ontario, Canada

Therapy based rehabilitation services $v$ no routine input in community dwelling patients who have had a stroke*

\begin{tabular}{lllllll}
\hline \multirow{2}{*}{$\begin{array}{l}\text { Outcomes at } \\
\text { 4-12 months }\end{array}$} & Number of trials $(\mathbf{n})$ & \multicolumn{3}{c}{ Weighted event rates } & & Rehabilitation \\
\hline Deterioration & $11(1350)$ & $30.5 \%$ & No routine input & RRR (95\% Cl) & NNT (CI) \\
\hline
\end{tabular}

*Deterioration = decrease in activities of daily living scores, dependency, or death. Abbreviations defined in glossary; RRR, NNT, Cl, and weighted event rates calculated from data in article using a fixed effects model. 